\title{
Is fecal calprotectin always normal in children with irritable bowel syndrome?
}

\author{
You Jin Choi ${ }^{1}$, Su Jin Jeong ${ }^{2}$ \\ ${ }^{1}$ Department of Pediatrics, Inje University Ilsan Paik Hospital, Goyang, ${ }^{2}$ Department of Pediatrics, CHA Bundang Medical Center, \\ CHA University School of Medicine, Seongnam, Korea
}

Background/Aims: Fecal calprotectin (FC) is a marker of intraluminal intestinal inflammation. Intestinal inflammation may contribute to the pathophysiology of irritable bowel syndrome (IBS). This study evaluated FC levels in children with IBS and differences in FC levels in children stratified by IBS subtype and healthy controls (HCs). Methods: A total of 157 children with IBS and 56 HCs aged 4-16 years (119 boys, 94 girls, mean age of 9.48 years) were included in this prospective study. Children with IBS were diagnosed using the Rome III criteria and classified into 4 subtypes: IBS with constipation (IBS-C, $n=37$ ), IBS with diarrhea (IBS-D, $\mathrm{n}=54$ ), IBS with alternating constipation and diarrhea (IBS-M, $\mathrm{n}=49$ ), and IBS unsubtyped (IBS-U, $\mathrm{n}=17$ ); postinfectious IBS (PI-IBS) was also considered. The FC concentration in stool samples was analyzed using an enzyme-linked immunosorbent assay. All participants answered a questionnaire regarding several demographic and clinical characteristics. Results: Children with IBS had significantly higher levels of FC than the HCs $(88.71 \mu \mathrm{g} / \mathrm{g}$ vs. $17.77 \mu \mathrm{g} / \mathrm{g})$. Among the 4 IBS subtypes, the FC concentration was highest in children with IBS-D, followed by those with IBS-M, IBS-C, and IBS-U ( $169.94 \mu \mathrm{g} / \mathrm{g}$ vs. $45.04,31.22$, and $33.52 \mu \mathrm{g} / \mathrm{g}$, respectively), and these differences were statistically significant. For PI-IBS, $90 \%$ of cases were in the IBS-D group. Conclusions: The FC level was significantly higher in children with IBS than in HCs and differed depending on the IBS subtype, supporting the notion that IBS is a type of low-grade bowel inflammation. (Intest Res 2019;17:546-553)

Key Words: Calprotectin; Irritable bowel syndrome; Postinfectious irritable bowel syndrome; Childhood

\section{INTRODUCTION}

Irritable bowel syndrome (IBS) during childhood is a common and chronic functional GI disorder that affects quality of life and has a prevalence of $2 \%$ to $24 \%$ worldwide. ${ }^{1}$ IBS is characterized by abdominal pain or discomfort associated with changing bowel habits, including the frequency of defecation and stool consistency, in the absence of an organic disease. ${ }^{2}$

The pathophysiology of IBS is not completely understood; however, dysregulation of the brain-gut axis is believed to lead to IBS. ${ }^{3}$ Various central and peripheral mechanisms are indi-

Received January 22, 2019. Revised July 21, 2019. Accepted July 22, 2019. Correspondence to Su Jin Jeong, Department of Pediatrics, CHA Bundang Medical Center, CHA University School of Medicine, 59 Yatap-ro, Bundanggu, Seongnam 13496, Korea. Tel: +82-31-780-5230, Fax: +82-31-780-5239, E-mail:jinped@cha.ac.kr cated to be involved, such as altered GI motility, visceral hypersensitivity, alternation in the intestinal microbiota, lowgrade immune activation, and intestinal inflammation, which may be linked to enhanced exposure to antigens due to increased intestinal permeability. ${ }^{3-7}$ Therefore, IBS is a very heterogeneous disorder and can be subtyped based on the bowel habits of the patients, such as diarrhea or constipation. ${ }^{8}$ Furthermore, some of the organic mechanisms of IBS are partly shared with the pathophysiology of IBD, and similarities in clinical manifestations are also observed between IBS and IBD. ${ }^{3,9}$ Postinfectious IBS (PI-IBS), which is diagnosed on the basis of acute onset of symptoms meeting the diagnostic criteria for IBS, has been regarded as a typical condition resulting from intestinal inflammation accompanied by other various pathophysiologic mechanisms. ${ }^{10}$

Calprotectin is a calcium-binding protein found in neutro- 
phils, monocytes, and macrophages. Fecal calprotectin (FC) reflects the increased migration of neutrophils due to inflammation of the intestinal mucosa. ${ }^{11-13} \mathrm{FC}$ is a useful marker to quantify intestinal inflammation, and FC concentrations are significantly increased in patients with IBD. The correlation of FC with clinical disease activity helps distinguish IBD from noninflammatory or functional bowel conditions such as IBS with high sensitivity and specificity. ${ }^{12-16}$ Currently, FC concentrations $<50 \mu \mathrm{g} / \mathrm{g}$ are considered normal. ${ }^{17} \mathrm{FC}$ concentrations greater than $200 \mu \mathrm{g} / \mathrm{g}$ measured with high sensitivity and specificity are considered to indicate the presence of an endoscopically active state of IBD. ${ }^{11,15}$ However, no publications directly comparing FC concentrations among different types of patients with IBS, including children and adults, are available.

The aims of this study were to evaluate differences in the FC concentration and the presence of PI-IBS among pediatric patients with 4 subtypes of IBS and healthy controls (HCs).

\section{METHODS}

\section{Study Participants}

This prospective study was performed in the Department of Pediatric Gastroenterology, Bundang CHA Medical Center, South Korea. We included a total of 172 children (73 girls and 99 boys) aged 4 to 16 years who were diagnosed with IBS using the pediatric Rome III criteria for IBS $^{17,18}$ and visited the Department of Pediatric Gastroenterology, Bundang CHA
Medical Center between January 1, 2014, and December 31, 2016. In addition, 56 healthy children of the same age group who participated as volunteers for this study were recruited during the same period and assigned to the control group. All participants and their parents or guardians answered a questionnaire regarding several demographic and clinical characteristics, such as the frequency of defecation and stool consistency. Children or their caregivers (in cases of children who were not yet able to read and write) kept a 2-week pain and stool diary. Abdominal pain or discomfort ratings were determined 4 times per day (awakening, after lunch, evening, and sleep) during the 2-week period.

The exclusion criteria included several conditions that could influence the movement or environment of the GI tract, for example, acute gastroenteritis, other GI tract disorders such as gastroesophageal reflux, Helicobacter pylori infection, or colon polyps, and the use of antibiotics, probiotics, NSAIDs, or steroids within 1 month of stool sampling, as well as a history of abdominal surgery or other congenital conditions. In addition, we excluded children with a history of preterm birth (gestational age $<36$ weeks), low or high birth weight or a small or large percentile of weight for age $(<3$ rd percentile or $>97$ th percentile) or those who had positive stool results for a viral or bacterial infection confirmed via PCR or stool culture. In total, 11 patients were excluded, and 4 did not provide stool samples.

Finally, stool samples ( $\mathrm{n}=213,94$ girls and 119 boys) were obtained from 157 children with IBS and 56 HCs (Fig. 1). This

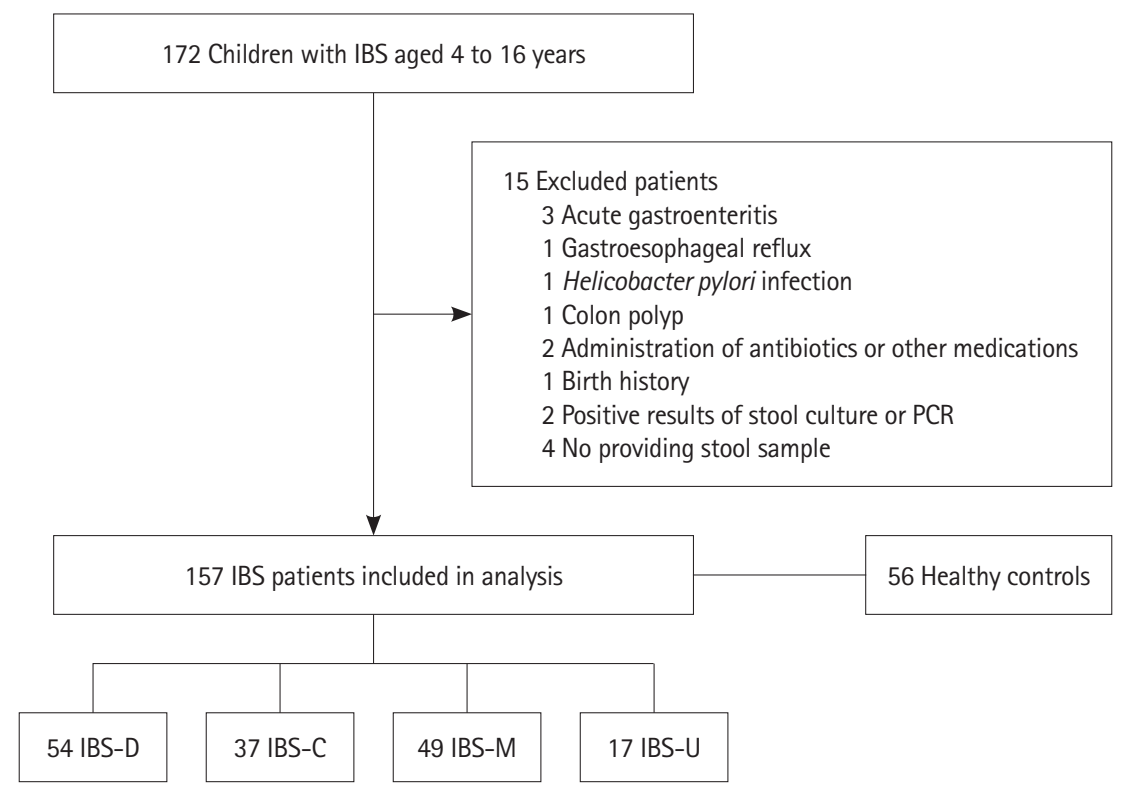

Fig. 1. Selection of the study participants. IBS-D, IBS with diarrhea; IBS-C, IBS with constipation; IBS-M, IBS with alternating constipation and diarrhea; IBS-U, IBS unsubtyped. 
Table 1. Rome III Diagnostic Criteria for IBS in Childhood and Subtyping of IBS

\author{
Rome III criteria for IBS in childhood \\ All of the following: \\ 1. Abdominal discomfort ${ }^{b}$ or pain associated with 2 or more of the following at least $25 \%$ of the time: \\ a. Improved with defecation \\ b. Onset associated with a change in the frequency of stool \\ c. Onset associated with a change in the form (appearance) of stool \\ 2. No evidence of an inflammatory, anatomic, metabolic, or neoplastic process that explains the subject's symptoms \\ Subtyping IBS by predominant stool pattern \\ 1. IBS with constipation-hard or lumpy stool ${ }^{c} \geq 25 \%$ and loose or watery stool ${ }^{d}<25 \%$ of bowel movements \\ 2. IBS with diarrhea-loose or watery stool $\geq 25 \%$ and hard or lumpy stool $<25 \%$ of bowel movements \\ 3. Mixed IBS-hard or lumpy stool $\geq 25 \%$ and loose or watery stools $\geq 25 \%$ of bowel movements \\ 4. Unsubtyped IBS
}

${ }^{a}$ Criteria fulfilled at least once per week for at least 2 months before diagnosis.

${ }^{b}$ An uncomfortable sensation not described as pain.

'Type I (small, hard, and lump patterns that look like goat feces) and type II (several hard lumps that look like goat feces and form clusters) from the Bristol Stool Scale.

${ }^{d}$ Type VI (fluffy pieces with ragged edges and mushy stool) and type VII (watery, no solid pieces, and entirely liquid) from the Bristol Stool Scale.

study was approved by the Ethical Committee of the Bundang CHA Medical Center (IRB No. BD2015-008). Written informed consent was obtained from the parents or guardians of the children participating, and a consent form was provided by all children aged 6 years and older.

\section{Subtypes of IBS and PI-IBS}

Classification of IBS into different subtypes was based on previous recommendations for IBS in adults as no pediatric Rome subtype criteria for children exist (Table 1) ${ }^{2,18}$ The stools from our patients were compared to the Bristol Stool Scale, a validated stool form scale that divides defecation into 7 grades according to stool cohesion and surface cracking and evaluates constipation. Patients with stools corresponding to type I (small, hard, and lump patterns that look like goat feces) and type II (several hard lumps that look like goat feces and form clusters) are considered to have constipation (hard stool). On the other hand, patients with stools corresponding to type VI (fluffy pieces with ragged edges, mushy stool) and type VII (watery, no solid pieces, entirely liquid) are considered to have diarrhea (loose stool). Patients were subtyped as having IBS-D (hard $<25 \%$ and loose $\geq 25 \%$ ), IBS-C (hard $\geq 25 \%$ and loose $<25 \%$ ), IBS-M (hard or loose $\geq 25 \%$ ) or IBS-U (unsubtyped). ${ }^{2}$

PI-IBS is defined as new acute onset of IBS following an episode of acute gastroenteritis characterized by any 2 of the following: diarrhea, vomiting, fever, and stool culture positivity for an infectious agent meeting the Rome III diagnostic crite- ria for IBS. ${ }^{10}$

\section{Stool Sample Collection and FC Measurement}

A stool sample weighing at least $5 \mathrm{~g}$ was collected into a sterile plastic container within 3 days of visiting us and was transferred to us on the same or next day and immediately processed. When the analyses could be performed after storage, the samples were stored frozen at $-20^{\circ} \mathrm{C}$. We measured $\mathrm{FC}$ quantitatively using a commercially available fluorescence enzyme immunoassay (FEIA; Green Cross Laboratories, Yongin, Korea). The supernatant of a centrifuged solution containing $0.2 \mathrm{~g}$ of stool was used to assess the calprotectin level using ImmunoCAP 250 (Phadia-Thermo Scientific, Uppsala, Sweden), with a lower detection limit of $11.5 \mu \mathrm{g} / \mathrm{g}$ and an upper detection limit of 2,000 $\mu \mathrm{g} / \mathrm{g}$.

To evaluate GI infection, a multiplex viral and bacterial stool PCR was performed for all the stool samples. Cases with a positive result were excluded. When the FC concentration was $>200 \mu \mathrm{g} / \mathrm{g}$, laboratory tests, including those for inflammatory markers such as CRP and ESR, and a colonoscopy with biopsies of the intestinal mucosa were performed within 1 or 2 weeks of receiving the results of initial stool sampling. The FC concentration was also measured 4 weeks after the initial fecal sampling without any medication use, and a number of IBS patients who had FC levels over $200 \mu \mathrm{g} / \mathrm{g}$ had no changes in their IBS subtypes during the 4 weeks, but the data were not included in the results of this study. 


\section{Statistical Analysis}

All statistical analyses were conducted using SPSS version 20 (IBM Corp., Armonk, NY, USA). Normally distributed data are shown as the mean \pm SD. For dichotomous data, differences in the baseline values between 2 groups were statistically tested using a chi-square test or Fisher exact test. For continuous data, the independent $t$-test or Mann-Whitney test and oneway ANOVA were carried out. FC concentrations are presented as the mean $\pm \mathrm{SD}$ and range. Multiple comparisons of the FC concentration between different patient groups and HCs were analyzed using a one-way ANOVA followed by the Dunnett test or Scheffe test and Kruskal-Wallis analysis. A $P$-value $<0.05$ was considered statistically significant.

\section{RESULTS}

We enrolled 157 children aged $4-16$ years who fulfilled the pediatric Rome III criteria for IBS (91 boys [57.9\%], 66 girls [42.1\%], mean age of $9.48 \pm 3.41$ years) and 56 HCs (28 boys [50\%], 28

Table 2. Baseline Characteristics and Fecal Calprotectin Concentrations of Children with IBS and HC

\begin{tabular}{lcc}
\hline Variable & IBS $(\mathrm{n}=157)$ & HC $(\mathrm{n}=56)$ \\
\hline Age (yr) & $9.48 \pm 3.41$ & $8.45 \pm 3.39$ \\
Boy & $91(57.9)$ & $28(50.0)$ \\
Race (Korean) & $153(97.5)$ & $54(96.4)$ \\
Living status (with 2 parents) & $147(93.6)$ & $51(91.0)$ \\
Education & & \\
$\quad$ School (8-16 yr) & $99(99.0)$ & $30(100.0)$ \\
$\quad$ Childcare (4-7 yr) & $47(82.5)$ & $20(76.9)$ \\
Duration of symptoms (mon) & $7.66 \pm 4.37$ & - \\
\hline
\end{tabular}

Values are presented as mean \pm SD or number (\%).

$\mathrm{HC}$, healthy control. girls [50\%], mean age of $8.45 \pm 3.39$ years). No significant difference was observed in age, sex and other baseline characteristics between the children with IBS and HCs (Table 2).

The mean concentration of FC was significantly higher in the children with IBS than in the HCs $(83.50 \pm 164.74$ and $17.77 \pm 10.92 \mu \mathrm{g} / \mathrm{g}, P<0.001)$. In the comparison of FC concentrations according to the subtypes of IBS (IBS-D, IBS-C, IBS-M, and IBS-U), the FC concentration in IBS-D stools had the highest mean value, followed by IBS-M, IBS-U, and IBS-C stools, and these differences were statistically significant (Table 3). In children with IBS-D ( $n=54)$, the mean FC concentration was $169.94 \mu \mathrm{g} / \mathrm{g}$, representing the highest concentration among all subtypes of IBS and the only concentration above the cutoff value $(50 \mu \mathrm{g} / \mathrm{g})$.

In addition, the highest FC concentration in the IBS-D subgroup was $1,243.7 \mu \mathrm{g} / \mathrm{g}$, which was the peak level in all participants. In contrast, the mean FC concentrations in children with IBS-M, IBS-C, and IBS-U were lower $(45.04,31.22$, and $33.52 \mu \mathrm{g} / \mathrm{g}$, respectively), below the normal cutoff level, and similar to the mean FC concentration in the HC group.

Fig. 2 shows the distributions of FC concentrations according to each subtype of IBS and in the HCs. In the HCs, the FC concentration did not rise above $50 \mu \mathrm{g} / \mathrm{g}$, which is the cutoff value for the normal FC level; the highest FC level among the HCs was $45.3 \mu \mathrm{g} / \mathrm{g}$. On the other hand, $34.4 \%$ of the children with IBS ( $\mathrm{n}=54)$ had an FC concentration above $50 \mu \mathrm{g} / \mathrm{g}$. Furthermore, $10.2 \%$ of the children with IBS ( $\mathrm{n}=16 ; 14$ IBS-D and 2 IBS-M) were found to have an FC concentration $>200 \mu \mathrm{g} / \mathrm{g}$, which is known to be related to the active phase of IBD. These 16 patients had no specific results with regard to medical history and laboratory evaluation except for high FC levels. As many as $55.6 \%(\mathrm{n}=30)$ of the children with IBS-D had an FC concentration $>50 \mu \mathrm{g} / \mathrm{g}$, and $25.9 \%(\mathrm{n}=14)$ had an FC concentration $>200 \mu \mathrm{g} / \mathrm{g}$. Among the children with IBS-C, 32.4\%

Table 3. Differences in the FC Concentration among Children with IBS and HCs

\begin{tabular}{|c|c|c|c|c|c|c|}
\hline \multirow{2}{*}{ Variable } & \multicolumn{4}{|c|}{ IBS $(n=157)$} & \multirow{2}{*}{$\begin{array}{c}\mathrm{HC} \\
(n=56)\end{array}$} & \multirow{2}{*}{$P$-value } \\
\hline & IBS-D $(n=54)$ & IBS-C $(n=37)$ & IBS-M $(n=49)$ & IBS-U $(n=17)$ & & \\
\hline No. of patients ( $>50 \mu \mathrm{g} / \mathrm{g}$ of FC) & $30(55.6)$ & $12(30.8)$ & $10(21.7)$ & $2(11.8)$ & 0 & \\
\hline Min-Max of FC $(\mu \mathrm{g} / \mathrm{g})$ & $11.5-1,243.7$ & $11.5-83.2$ & $11.5-258.5$ & $11.5-114.5$ & $11.5-45.3$ & \\
\hline \multirow[t]{2}{*}{ Mean of FC $(\mu \mathrm{g} / \mathrm{g})$} & $169.94 \pm 253.78$ & $31.22 \pm 23.23^{\mathrm{a}}$ & $45.04 \pm 56.26^{b}$ & $33.52 \pm 28.35^{b}$ & $17.77 \pm 10.92$ & $<0.001$ \\
\hline & \multicolumn{4}{|c|}{$83.50 \pm 164.74$} & & $<0.001$ \\
\hline
\end{tabular}

Values are presented as number (\%) or mean \pm SD.

${ }^{a} P<0.001,{ }^{b} P<0.01$ compared with IBS-D (Dunnett test).

FC, fecal calprotectin; HC, healthy control; IBS-D, IBS with diarrhea; IBS-C, IBS with constipation; IBS-M, IBS with alternating constipation and diarrhea; IBS-U, IBS unsubtyped; Min, minimum; Max, maximum. 


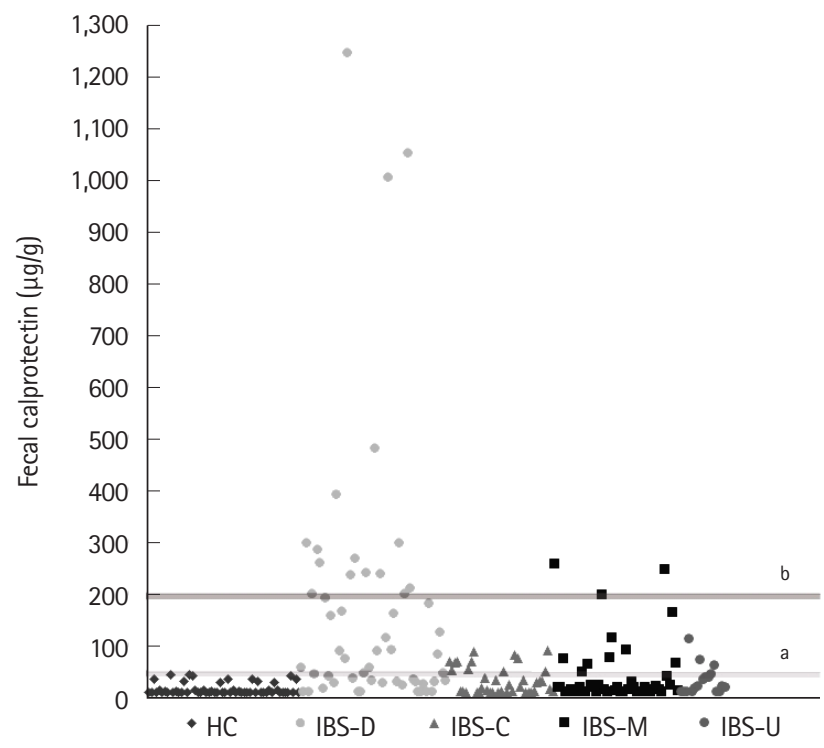

Fig. 2. The distributions of fecal calprotectin in IBS patients and healthy controls. ${ }^{a}$ Cutoff of fecal calprotectin, $50 \mu \mathrm{g} / \mathrm{g}$; ${ }^{\text {CC }}$ utoff of threshold value of active organic disease, $200 \mu \mathrm{g} / \mathrm{g}$. HC, healthy controls; IBS-D, IBS with diarrhea; IBS-C, IBS with constipation; IBS$M$, IBS with alternating constipation and diarrhea; IBS-U, IBS unsubtyped.

$(\mathrm{n}=12)$ had FC concentrations $>50 \mu \mathrm{g} / \mathrm{g}$; however, the maximum $\mathrm{FC}$ value was $91.6 \mu \mathrm{g} / \mathrm{g}$.

Of the children with IBS, $12.7 \%(n=20)$ had manifestations of PI-IBS. No significant differences in sex or age were found between the patients with PI-IBS and those with non-PI-IBS. The mean concentrations of FC in the patients with PI-IBS and non-PI-IBS were $368.24 \pm 332.64$ and $41.93 \pm 44.81 \mu \mathrm{g} / \mathrm{g}$, respectively. Table 4 shows the proportion of PI-IBS in each of the 4 IBS subtypes. Among our IBS patients with high FC concentrations ( $>200 \mu \mathrm{g} / \mathrm{g}), 93.8 \%(\mathrm{n}=15)$ had manifestations of PI-IBS, and all 3 patients in the IBS-D group with extremely high FC concentrations ( $>1,000 \mu \mathrm{g} / \mathrm{g})$ had manifestations of PI-IBS.

\section{DISCUSSION}

This study aimed to evaluate differences in the FC concentration and the presence of PI-IBS among pediatric patients with 4 subtypes of IBS and HCs. This is the first study to compare the FC concentration between patients with IBS and HCs and among the 4 IBS subtype (IBS-D, IBS-C, IBS-M, and IBS-U) in a pediatric population. In the present study, children with IBS had a higher FC concentration than the HCs. In particular, the mean FC concentration in the IBS-D group was above the
Table 4. PI-IBS in Each Subtype of IBS

\begin{tabular}{lccr}
\hline Variable & $\begin{array}{c}\text { PI-IBS } \\
(n=20)\end{array}$ & $\begin{array}{c}\text { Non-PI-IBS } \\
(n=137)\end{array}$ & $P$-value \\
\hline Age $(y r)$ & $10.75 \pm 3.08$ & $9.30 \pm 3.42$ & 0.075 \\
Boy & $15(75.0)$ & $76(55.4)$ & 0.098 \\
Fecal calprotectin $(\mu \mathrm{g} / g)$ & $368.24 \pm 332.64$ & $41.93 \pm 44.81$ & $<0.001$ \\
IBS subtype & & & \\
IBS-D $(n=54)$ & 18 & 36 & \\
IBS-C $(n=37)$ & 0 & 37 & \\
IBS-M $(n=49)$ & 2 & 47 & \\
IBS-U $(n=17)$ & 0 & 17 & \\
\hline
\end{tabular}

Values are presented as mean $\pm S D$, number $(\%)$, or number.

$\mathrm{PI}-\mathrm{IBS}$, postinfectious IBS; IBS-D, IBS with diarrhea; IBS-C, IBS with constipation; IBS-M, IBS with alternating constipation and diarrhea; IBS-U, IBS unsubtyped.

normal FC cutoff value and was higher than those in the HCs and children with other IBS subtypes. The IBS-D group also showed significant correlations with PI-IBS in contrast to the other subtypes of IBS.

Numerous controlled studies have shown that FC is a useful marker of intestinal inflammation, particularly because its concentration seems to be unaffected by inflammation outside the GI tract. ${ }^{11-13}$ The Buhlmann calprotectin assay yields the following threshold values for adults and children aged 4 to 17 years: normal value $<50 \mu \mathrm{g} / \mathrm{g}$; mild organic disease 50 $200 \mu \mathrm{g} / \mathrm{g}$; and active organic disease $>200 \mu \mathrm{g} / \mathrm{g} .{ }^{19}$ Although FC may also increase in other types of inflammation, such as colon cancer and bacterial inflammation, or when taking various medications such as NSAIDs or steroids, it correlates significantly with the endoscopic and histological activity of IBD. ${ }^{13}$ Therefore, pediatric gastroenterologists have been particularly interested in the use of FC as a diagnostic tool to distinguish organic disease from functional disorders, which may help minimize the use of invasive procedures such as colonoscopy in children. Several studies have investigated the FC concentration in patients with IBS to prove the applicability of FC as a diagnostic marker for distinguishing healthy individuals or patients with IBS from patients with IBD; FC concentrations in patients with suspicious or remised IBD were significantly higher than those in healthy individuals or patients with IBS.,14 However, in the present study, $34.4 \%$ of the children with IBS $(\mathrm{n}=41)$ had an FC concentration above the normal cutoff value $(50 \mu \mathrm{g} / \mathrm{g})$, and $10.2 \%$ of the children with IBS-D or IBS-M had very high FC concentrations $>200 \mu \mathrm{g} / \mathrm{g}$. This finding contrasts with the results of previously published studies in which 
both patients with IBS and HCs were shown to have FC concentrations below the cutoff value, unlike patients with IBD., ${ }^{9,12}$ Although studies of pediatric patients with functional IBS are abundant, few studies have investigated FC in the pediatric population. In particular, no research comparing FC concentrations between IBS patients and HCs has been performed in children/adolescents. However, a recent study reported that the mean FC level in adult patients with PI-IBS was significantly higher than the cutoff value and that in a control group without digestive symptoms $(90.5 \pm 180 \mu \mathrm{g} / \mathrm{g}$ vs. $24.3 \pm 16.1 \mu \mathrm{g} / \mathrm{g}$, $P<0.01){ }^{20}{ }^{20}$ The results of our study also showed that the mean FC concentration in the PI-IBS group was higher than $50 \mu \mathrm{g} / \mathrm{g}$. We remeasured the FC level in children with IBS who exhibited an FC $>200 \mu \mathrm{g} / \mathrm{g}$ after 4 weeks and found that the level had decreased to the normal range below the cutoff value, and these children were confirmed to have IBS rather than IBD by colonoscopy.

IBS is generally regarded as a multifactorial disorder involving a host and environmental factors. It is one of the most common functional GI disorders in both children and adults. ${ }^{6,8}$ The clinical symptoms or signs of IBS depend on the contributions of various factors, such as low-grade intraluminal inflammation, immune activation, enteric neuromuscular dysfunction, and brain-gut axis dysregulation, ${ }^{4-6}$ enabling categorization of IBS into several subtypes, including IBS-D, IBS-C, IBS-M, and IBS-U. ${ }^{8}$ Notably, impaired intestinal permeability due to altered tight junctions in the luminal mucosa or immune activation is connected with low-grade intestinal inflammation, which is associated with visceral hypersensitivity. This mechanism has recently been described and verified by several clinical studies on IBS and IBD. Impaired intestinal permeability was reported to be related to the severity of abdominal pain in patients with IBS and was more pronounced in IBS-D subtype patients than in patients with other subtypes of IBS ${ }^{5,6}$ and patients with PI-IBS. ${ }^{10}$ Furthermore, patients with IBS with impaired intestinal permeability were more likely to report increased stool frequency. ${ }^{21}$

In many cases, patients with IBS and IBD share subtle symptoms such as abdominal pain, diarrhea, and constipation; therefore, clinicians experience difficulty in distinguishing between IBS and IBD. ${ }^{14,22}$ In the present study, the patients with a very high concentration of FC $(>200 \mu \mathrm{g} / \mathrm{g})$ were confirmed to have IBS and not IBD by remeasurement of the FC concentration and by colonoscopy. The low-grade chronic intestinal inflammation observed in IBS is also observed in IBD. The results of this investigation provide evidence that low-grade chronic intestinal inflammation and alterations in intestinal permeability play roles in the pathogenic pathways of IBS via FC. FC is the most sensitive marker of the intestinal state; the concentration of FC in some children with IBS in the present study, especially those with IBS-D or PI-IBS, was increased as a consequence of these pathologies.

This study has a few limitations. First, the sample size was relatively small for comparisons of each subtype of IBS with the HC group. Furthermore, although intestinal dysbiosis and altered intestinal permeability are considered critical mechanisms in intestinal inflammation, ${ }^{9,23}$ we could not analyze the fecal microbiota from our fecal samples and did not evaluate intestinal permeability. Our contrasting results are likely to show some novel features of childhood IBS, despite the small sample size. Although there are many pediatric patients with functional IBS, few FC studies have been performed in the pediatric population. Particularly, no study has looked at IBS and $\mathrm{HC}$ in children/adolescents. To resolve the above issues, further studies with larger sample sizes and long-term follow-ups of children with IBS with high FC concentrations that are based on recent diagnostic criteria are required. Therefore, we are further assessing the FC levels in these IBS patients and reviewing their clinical courses to prepare a larger report on these findings. IBS subtyping is considered as an essential part of future research, and it could be influenced by various individual and environmental characteristics or interactions with the microbiota in the intestine as children grow toward adulthood. Studies of pediatric IBS should evaluate more than just FC levels and the microbiome.

In conclusion, FC is known to serve as an appropriate marker to distinguish IBS from organic diseases such as IBD; however, we found that some pediatric patients with IBS had a high FC concentration similar to that observed in patients with IBD, particularly those with the IBS-D subtype or PI-IBS These patients require more detailed examinations, close observation, and follow-up than do those without high FC levels. Furthermore, IBS pediatric patients with high FC levels and their caregivers are more likely to agree with further more invasive evaluations, if warranted.

Therefore, in clinical practice, we suggest that the FC concentration should be measured in pediatric patients with IBS and in those with an FC concentration above the normal cutoff value. The clinical symptoms of IBS-D or PI-IBS should be clarified and evaluated, and FC should be measured repeatedly at stated intervals. 


\section{FINANCIAL SUPPORT}

The authors received no financial support for the research, authorship, and/or publication of this article.

\section{CONFLICT OF INTEREST}

No potential conflict of interest relevant to this article was reported.

\section{AUTHOR CONTRIBUTION}

Conceptualization: Jeong SJ. Methodology: Choi YJ, Jeong SJ. Formal analysis: Choi YJ, Jeong SJ. Project administration: Jeong SJ. Visualization: Choi YJ. Writing - original draft: Choi YJ, Jeong SJ. Writing - review and editing: Choi YJ, Jeong SJ. Approval of the final manuscript: all authors.

\section{ORCID}

Choi YJ

Jeong SJ

https://orcid.org/0000-0002-6882-3877

https://orcid.org/0000-0002-7388-8368

\section{REFERENCES}

1. Devanarayana NM, Rajindrajith S, Pathmeswaran A, Abegunasekara C, Gunawardena NK, Benninga MA. Epidemiology of irritable bowel syndrome in children and adolescents in Asia. J Pediatr Gastroenterol Nutr 2015;60:792-798.

2. Longstreth GF, Thompson WG, Chey WD, Houghton LA, Mearin F, Spiller RC. Functional bowel disorders. Gastroenterology 2006;130:1480-1491.

3. Abdul Rani R, Raja Ali RA, Lee YY. Irritable bowel syndrome and inflammatory bowel disease overlap syndrome: pieces of the puzzle are falling into place. Intest Res 2016;14:297-304.

4. von Arnim U, Wex T, Ganzert C, Schulz C, Malfertheiner P. Fecal calprotectin: a marker for clinical differentiation of microscopic colitis and irritable bowel syndrome. Clin Exp Gastroenterol 2016;9:97-103.

5. Gecse K, Róka R, Ferrier L, et al. Increased faecal serine protease activity in diarrhoeic IBS patients: a colonic lumenal factor impairing colonic permeability and sensitivity. Gut 2008;57:591-599.

6. Barbara G, Zecchi L, Barbaro R, et al. Mucosal permeability and immune activation as potential therapeutic targets of probiotics in irritable bowel syndrome. J Clin Gastroenterol
2012;46:S52-S55.

7. Di Nardo G, Barbara G, Cucchiara S, et al. Neuroimmune interactions at different intestinal sites are related to abdominal pain symptoms in children with IBS. Neurogastroenterol Motil 2014;26:196-204.

8. Saha L. Irritable bowel syndrome: pathogenesis, diagnosis, treatment, and evidence-based medicine. World J Gastroenterol 2014;20:6759-6773.

9. Keohane J, O’Mahony C, O’Mahony L, O’Mahony S, Quigley EM, Shanahan F. Irritable bowel syndrome-type symptoms in patients with inflammatory bowel disease: a real association or reflection of occult inflammation? Am J Gastroenterol 2010;105:1788-1794.

10. Spiller R, Garsed K. Postinfectious irritable bowel syndrome. Gastroenterology 2009;136:1979-1988.

11. von Roon AC, Karamountzos L, Purkayastha S, et al. Diagnostic precision of fecal calprotectin for inflammatory bowel disease and colorectal malignancy. Am J Gastroenterol 2007;102:803813.

12. Menees SB, Powell C, Kurlander J, Goel A, Chey WD. A metaanalysis of the utility of C-reactive protein, erythrocyte sedimentation rate, fecal calprotectin, and fecal lactoferrin to exclude inflammatory bowel disease in adults with IBS. Am J Gastroenterol 2015;110:444-454.

13. Alibrahim B, Aljasser MI, Salh B. Fecal calprotectin use in inflammatory bowel disease and beyond: a mini-review. Can J Gastroenterol Hepatol 2015;29:157-163.

14. Schoepfer AM, Trummler M, Seeholzer P, Seibold-Schmid B, Seibold F. Discriminating IBD from IBS: comparison of the test performance of fecal markers, blood leukocytes, CRP, and IBD antibodies. Inflamm Bowel Dis 2008;14:32-39.

15. Montalto M, Gallo A, Santoro L, D'Onofrio F, Landolfi R, Gasbarrini A. Role of fecal calprotectin in gastrointestinal disorders. Eur Rev Med Pharmacol Sci 2013;17:1569-1582.

16. Olafsdottir I, Nemeth A, Lörinc E, Toth E, Agardh D. Value of fecal calprotectin as a biomarker for juvenile polyps in children investigated with colonoscopy. J Pediatr Gastroenterol Nutr 2016;62:43-46.

17. Waugh N, Cummins E, Royle P, et al. Faecal calprotectin testing for differentiating amongst inflammatory and non-inflammatory bowel diseases: systematic review and economic evaluation. Health Technol Assess 2013;17:1-211.

18. Self MM, Czyzewski DI, Chumpitazi BP, Weidler EM, Shulman RJ. Subtypes of irritable bowel syndrome in children and adolescents. Clin Gastroenterol Hepatol 2014;12:1468-1473.

19. Labaere D, Smismans A, Van Olmen A, et al. Comparison of six 
different calprotectin assays for the assessment of inflammatory bowel disease. United European Gastroenterol J 2014;2:3037.

20. Melchior C, Aziz M, Aubry T, et al. Does calprotectin level identify a subgroup among patients suffering from irritable bowel syndrome? Results of a prospective study. United European Gastroenterol J 2017;5:261-269.

21. Marshall JK, Thabane M, Garg AX, et al. Intestinal permeability in patients with irritable bowel syndrome after a water- borne outbreak of acute gastroenteritis in Walkerton, Ontario. Aliment Pharmacol Ther 2004;20:1317-1322.

22. Jonefjäll B, Strid H, Ohman L, Svedlund J, Bergstedt A, Simren M. Characterization of IBS-like symptoms in patients with ulcerative colitis in clinical remission. Neurogastroenterol Motil 2013;25:756-e578.

23. Saulnier DM, Riehle K, Mistretta TA, et al. Gastrointestinal microbiome signatures of pediatric patients with irritable bowel syndrome. Gastroenterology 2011;141:1782-1791. 